\title{
Defensa en profundidad para la evaluación de riesgos laborales por ruido en termoeléctrica
}

\section{Deep defense to asses labor risks for noise in power station}

\author{
Roani Ladislá Miranda Cuéllar', Manuel Perdomo Ojeda 2, Jesús Salomón Llanes ${ }^{2}$ \\ 1. Instituto Superior Politécnico José Antonio Echeverría. Habana. Cuba \\ 2. Instituto Superior de Tecnologías y Ciencias Aplicadas. Habana. Cuba. \\ Recibido: 3-06-15 \\ Aceptado: $31-08-15$

\section{Correspondencia} \\ Roani Ladislá Miranda \\ 114 \# 11901 e/ Ciclo Vía y Rotonda, Marianao 19390 Habana, Cuba \\ Facultad de Ingeniería Industrial \\ Instituto Superior Politécnico "José Antonio Echeverría" \\ Telef.: 7266-3531 \\ Correo electrónico: rmiranda@ind.cujae.edu.cu
}

Resumen

Introducción: Las técnicas de evaluación de riesgos laborales se llevan a cabo por diferentes procedimientos, a través de las aplicaciones de métodos normativos del cumplimiento de los parámetros asociados con las mediciones de los umbrales de daño a la salud del hombre. Sin embargo, no se tienen en cuenta los principios básicos de seguridad, que incluyen las experiencias de buenas prácticas en industrias de altos requisitos de seguridad y disponibilidad.

Objetivos: El objetivo de este artículo es proponer un método bajo el enfoque de defensa en profundidad para evaluar los riesgos laborales por ruido en la Central Termoeléctrica (CTE) de 100 MW.

Material y Métodos: Se aplicó la técnica de entrevistas a técnicos y especialistas en seguridad y salud del trabajo, así como tecnólogos para explorar los elementos que tomaban en cuenta en la evaluación de los riesgos laborales por ruido, y se realizó la consulta a especialistas para precisar los elementos que debían formar parte de los aspectos de seguridad de acuerdo al método propuesto. Además Se realizó la evaluación de los riesgos laborales por ruido de acuerdo a dos metodologías implementadas con anterioridad y la propuesta.

Resultados: Se muestran de acuerdo a las tres metodologías. Se determinó que la generación de ruidos no es un aspecto significativo a tener en cuenta, para la metodología 1, se evidenció una dispersión del nivel sonoro, con tendencia estable a los valores máximos en determinadas áreas según la Norma Cubana de ruido, para la metodología 2 mientras que para la metodología 3, los aspectos de seguridad evaluados negativamente por cada escalón, son los que se requieren tomar medidas correctivas dirigidas a modificar los ítems calificados negativamente a positivos para elevar el nivel de seguridad.

Conclusiones: La evaluación empleando escalones de defensa en profundidad aplicado a los riesgos laborales posibilita un análisis con el enfoque de procesos, que permite estimar la contribución relativa y sus causas, así como resolverlas en un corto plazo.

Med Segur Trab (Internet) 2015; 61 (240) 354-366

Palabras Clave: riesgos laborales por ruido, evaluación por escalones de defensa. 


\section{Abstract}

Abstract: The labour risks evaluation techniques are carried out using several methods, through the application of normative methods applying the execution of the parameters associated with the measurements of the men's health damage thresholds. Basic security principles are nevertheless not taken into account, which enclose the good practices experiences in high-requirement security and availability industries.

Objectives: The purpose of this article is to propose a method attending on the defence in depth in order to evaluate the noise labour risks in the Thermal Power Station (TPS) of 100 MW.

Material and Methods: In order to explore the elements considered in the evaluation of the noise labours risks, interviews to technicians and specialists in labour security and health were taken. It was carried out the consultation to specialists in order to specify the elements that should be part of the security aspects according to the nominative method. The evaluation of the labours risks for noise together with the proposal were also carried out according to two methodologies prior employed.

Results: three methodologies were used. It was determined that the noise generation is not a significant aspect to keep in mind for the methodology 1 . For the methodology 2 it was demonstrated a dispersion of the noise level with stable tendency to the maximal values in determining areas according to the Noise Cuban Norm, while for the methodology 3, the security aspects negatively evaluated for each step, are those which require to take corrective measures implemented to modify the negatively-qualified items to positive in order to raise the security level.

Conclusions: The evaluation using the depth security levels applied to the labour risks allows to estimate an analysis with the process approach, that allow not only to esteem the relative contribution and its causes but to resolve them in a short-term period.

Med Segur Trab (Internet) 2015; 61 (240) 354-366

Keywords: noise labours risks, evaluation of security levels. 


\section{INTRODUCCIÓN}

La implementación del Sistema de la Seguridad y Salud en el Trabajo se lleva a cabo en todo el mundo, con el objetivo de contribuir a la protección de los trabajadores siguiendo las directrices erigidas a nivel internacional y con la implementación de las normas OSHAS ${ }^{1}$, que en Cuba se establecen en la norma cubana de la serie $1800{ }^{2}$.

En el ambiente laboral en ocasiones se propician situaciones riesgosas para los trabajadores y están contempladas dentro de la salud laboral, existiendo diferentes clasificaciones de los riesgos profesionales o laborales que en sentido general convergen; asimismo hay varias formas de cuantificar los riesgos y elementos generales a tener en cuenta en la prevención de riesgos.

El Sistema de Seguridad y Salud del Trabajo está presente en cada empresa cubana y el estado brinda especial atención, fundamentalmente en empresas como las de generación de electricidad que a pesar de sus incuestionables beneficios, produce afectaciones a la salud humana, entre los que se encuentran los riesgos laborales por ruido.

En Cuba la salud ocupacional se sitúa en un lugar primordial y está respaldada por la voluntad política y jurídica del Estado, que tomando como base el medioambiente y en particular los riesgos profesionales, la República de Cuba es Estado parte del Convenio sobre la protección de los trabajadores contra los riesgos profesionales debidos a la contaminación del aire, el ruido y las vibraciones en el lugar de trabajo.

La legislación ambiental cubana constituye un importante instrumento de la política que se compone tanto de normas legales de diferentes jerarquías jurídicas como normas técnicas cubanas. A su vez, de acuerdo a la incidencia que las mismas tengan en la regulación de la temática ambiental se clasifica en legislación propiamente ambiental, tal es el caso de la Ley 81 "Del Medio Ambiente", o de relevancia ambiental sectorial como la Ley N. 13 de Protección e Higiene del Trabajo.

El marco legal en Cuba, con respecto a la seguridad y salud en el trabajo, tiene una estructura concatenada comenzando por Constitución de la República de Cuba (1976) (Derecho laboral: Capítulo VII: Derechos, deberes y garantías fundamentales. Artículo 48: El Estado garantiza el derecho a la protección, seguridad e higiene del trabajo, mediante la adopción de medidas adecuadas para la prevención de accidentes y enfermedades profesionales), y apoyado en el Acuerdo (s/n) del Consejo de Ministros de 1964 donde se establecen las Bases generales de la organización de la Protección e Higiene del Trabajo, que se precisan tanto en la Ley N. ${ }^{\circ} 13$ de Protección e Higiene del Trabajo como en su reglamento, que se encuentra establecido en el Decreto Ley N. ${ }^{\circ} 101$ de 1982 (Reglamento General de la Ley N. 13 de Protección e Higiene del Trabajo), como en la Resolución 39/2007, vinculado con las obras o actividades que deben ser sometidas al proceso de evaluación mediante la Resolución N. 31 del Ministerio de Trabajo y Seguridad Social (2002) (Sobre la identificación, evaluación y control de los factores de riesgo en el trabajo) y con la Resolución Conjunta 2/96 MINSAP-MTSS. Además se establece la metodología general para la identificación, evaluación y gestión de la prevención de los riesgos que afectan la seguridad y salud de los trabajadores ${ }^{3}$ y un conjunto de normas complementarias que establecen los parámetros permisibles, tal como precisa la norma cubana (NC) NC 19-01-04:1980 (SNPHT). Ruido. Requisitos generales higiénicos sanitarios), teniendo en cuenta la metodología general para la evaluación de la salud ocupacional.

Para la identificación de riesgos, se aplican métodos y técnicas, en industrias y procesos, tales como: análisis histórico, Failure análisis preliminar de riesgos, Mode and Effects Análisis (FMEA) ${ }^{4-7}$; y con combinaciones ${ }^{8}$, HAZOP ${ }^{9-11}$, y su visión social ${ }^{12}$; What if? (¿Qué pasa si?), entre otros. También se diseñan otros métodos y combinaciones para identificar los riesgos en los procesos ${ }^{13}$ y se crean software para agilizar el trabajo como en ${ }^{14}$. 
Estos métodos y técnicas se clasifican generalmente como cuantitativos o cualitativos. La legislación vigente establece guías y normativas, permitiendo personalizarlos para cada organización en particular. Sin embargo, en estas técnicas no se tienen en cuenta los principios básicos de seguridad, que incluyen las experiencias de buenas prácticas en industrias de altos requisitos de seguridad y disponibilidad.

La emisión de ruidos es una problemática inherente al proceso de generación de electricidad, pero afecta negativamente a la salud de los trabajadores vinculados directamente a la producción y en sus áreas aledañas, constituyendo un riesgo laboral. Las técnicas de evaluación de riesgos laborales, tradicionalmente, realizan mediciones de los umbrales de daño a la salud del hombre, mediante mediciones in situ y su comparación con los límites establecidos en dichas normas.

Este artículo presenta un método para la evaluación de los riesgos laborales por ruido en una (CTE) de $100 \mathrm{MW}$, bajo el enfoque de defensa en profundidad, que es semicualitativo.

El objetivo de este artículo es proponer un método bajo el enfoque de defensa en profundidad para evaluar los riesgos laborales por ruido en la Central Termoeléctrica (CTE) de $100 \mathrm{MW}$.

\section{MATERIAL Y MÉTODOS}

Para el diseño del método se tuvieron en cuenta los criterios que existen de la evaluación de los riesgos laborales por ruido en el ámbito nacional e internacional, así como la existencia de guías para llevar a cabo la implementación de los elementos del manejo de la seguridad y los cambios, en los procesos de investigación de incidentes, accidentes, planeación de emergencias y la respuesta ${ }^{15}$.

Se analizó la evaluación de los riesgos laborales por ruido en la Central Termoeléctrica (CTE) objeto de estudio, aplicándose la Guía metodológica para la implementación del Sistema de Gestión Ambiental en las entidades de la UNE y la Metodología para la identificación, evaluación y gestión de la prevención de los riesgos que afectan la seguridad y salud de los trabajadores contenida en el Manual de Procedimientos Seguridad y Protección UI-PS 0008.

Se aplicó la técnica de entrevistas a técnicos y especialistas en seguridad y salud del trabajo, así como tecnólogos para explorar los elementos que tomaban en cuenta en la evaluación de los riesgos laborales por ruido, así como la consulta a especialistas para precisar los elementos que debían formar parte de los aspectos de seguridad de acuerdo al método propuesto y se empleó el software ASeC ${ }^{16}$.

Se realizó la evaluación de los riesgos laborales por ruido de acuerdo a dos metodologías, la Guía metodológica para la implementación del Sistema de Gestión Ambiental en las entidades de la UNE y la Metodología para la identificación, evaluación y gestión de la prevención de los riesgos que afectan la seguridad y salud de los trabajadores contenida en el Manual de Procedimientos Seguridad y Protección UI-PS 0008, para compararlas con el método propuesto.

A continuación se muestran las evaluaciones efectuadas empleando cada método.

\section{Evaluación de los riesgos para la implementación del Sistema de Gestión Ambiental en las entidades de la UNE}

Para llevar a cabo esta metodología, se definen los aspectos ambientales de acuerdo al local o instalación y en dependencia de la actividad o proceso que se realiza. Los aspectos ambientales se han identificado a partir de las entradas y salidas de los procesos que se desarrollan en cada una de las instalaciones de la CTE, así como de los 
posibles riesgos que pueden ocurrir con afectación al medio ambiente, durante su ejecución.

Esta metodología establece criterios y escalas de evaluación que son mostrados en la tabla siguiente.

Tabla 1. Indicadores de evaluación de los aspectos ambientales de acuerdo a Guía metodológica para la implementación de un Sistema de Gestión Ambiental

\begin{tabular}{cll}
\hline No & \multicolumn{1}{c}{ Criterio } & \multicolumn{1}{c}{ Escala de evaluación } \\
\hline 1 & Severidad (cuán severo es el impacto que se produce, teniendo & 4 Extremadamente Severo \\
& en cuenta no solo las características del aspecto sino las & 3 Severo \\
& condiciones del medio receptor) & 2 Medio \\
& & 1 Ligero \\
& & 0 Despreciable \\
\hline 2 & Frecuencia (con qué frecuencia se produce el hecho que da & 4 Continuo \\
& lugar al impacto) & 3 Frecuente \\
& & 2 Algunas veces (irregular) \\
& & 1 Ocurre accidentalmente \\
\hline 3 & Efecto sobre la comunidad o los Trabajadores (magnitud del & 4 Muy alto \\
& efecto que el hecho produce o produciría potencialmente sobre & 3 Alto \\
& la comunidad o sobre los trabajadores) & 2 Mediano \\
& & 1 Bajo \\
& & 0 No detectable \\
\hline 4 & Sujeto a regulación (si el aspecto que se evalúa está sujeto a & 5 Regulado \\
& alguna regulación o reglamentación ambiental) & 0 No regulado \\
\hline
\end{tabular}

Se considerarán aspectos significativos, aquellos cuya evaluación total obtenida a partir de la suma de las evaluaciones de cada uno de los criterios considerados, sea $\geq 5$.

De acuerdo a la evaluación, se presenta el inventario de todos los aspectos ambientales identificados y sus impactos, señalando las actividades o procesos a los cuales están relacionados; se procede a la evaluación de cada aspecto, en correspondencia con la metodología. Para cada uno se define si ocurre durante el proceso de iteración normal, anormal o de accidente y se propone la medida o acción fundamental que debe acometerse respecto a cada aspecto para su control y manejo adecuado.

\section{Evaluación de los riesgos de acuerdo al Manual de Procedimientos Seguridad y Protección}

El Manual de Procedimientos Seguridad y Protección UI-PS 0008, de la Unión Eléctrica, del Ministerio de la Industria y Minas establece la metodología para la identificación, evaluación y gestión de la prevención de los riesgos que afectan la seguridad y salud de los trabajadores, así como diseñar e implantar las medidas preventivas que garanticen la seguridad y salud de los trabajadores.

La etapa inicial es el proceso de identificación de los riesgos, tomando como base la Resolución del Ministerio de Trabajo y Seguridad Social, en este análisis se tendrá en cuenta los documentos rectores en materias de seguridad, y las normas cubanas (NC) de ruido, así como los resultados de las inspecciones de la Técnica de Seguridad Industrial en las diferentes inspecciones y auditorías.

Los riesgos se evalúan cualitativamente asociados a su descripción presentes en las máquinas, puestos de trabajo, accesos, local, equipos de protección, entre otros y se asocia al grado de peligro; que se clasifican en peligro, peligro grave, nivel de acción y nivel de seguridad. El nivel de acción es cuando se está próximo a los valores admitidos según la norma y el nivel de seguridad es dentro de ese rango. Cuando los valores 
admitidos se sobrepasan según la norma es peligro y peligro grave, si está muy por encima de esos valores.

Se identificaron los riesgos por ruido que pueden existir por áreas y de acuerdo a las actividades que se realizan.

Además se define que la identificación y evaluación de riesgos se revisará y actualizará con una periodicidad de tres años o en las situaciones siguientes:

- Cuando se modifiquen los objetos, medios, procesos, operaciones, mecanismos de control del sistema de trabajo.

- Cuando ocurran incendios, explosiones, accidentes, averías o incidentes.

- En ocasión de la elección y adquisición de equipos, maquinarias o productos químicos, así como en la instalación de los mismos.

Sin embargo, no existe un acápite especial para los ruidos como enfermedad profesional existente que monitoree una pérdida auditiva significativa, tanto por la cantidad de trabajadores como por el nivel de incidencia.

De acuerdo a esta metodología existe una guía para definir las actuaciones derivadas de posibles conclusiones sobre los riesgos que se muestra en la tabla siguiente:

Tabla 2. Actuaciones derivadas de posibles conclusiones sobre los riesgos

\begin{tabular}{|c|c|}
\hline CONCLUSIONES & ACTUACIONES \\
\hline $\begin{array}{l}\text { Riesgos insignificantes en la actualidad, es poco } \\
\text { previsible que puedan aumentar en el futuro. }\end{array}$ & $\begin{array}{l}\text { Concluir la evaluación en esta etapa. No son } \\
\text { necesarias otras medidas. }\end{array}$ \\
\hline $\begin{array}{l}\text { Los riesgos se controlan dentro de un nivel } \\
\text { aceptable, por ejemplo: Con arreglo a normas } \\
\text { nacionales o ramales. }\end{array}$ & $\begin{array}{l}\text { Mejorar la protección si es posible. Acabar la } \\
\text { evaluación. } \\
\text { Seguimiento de las medidas de control. }\end{array}$ \\
\hline $\begin{array}{l}\text { Los riesgos se controlan en la actualidad, } \\
\text { posiblemente podrían aumentar en el futuro, porque } \\
\text { los sistemas de control actuales pueden comenzar a } \\
\text { fallar o a ser utilizados incorrectamente. }\end{array}$ & $\begin{array}{l}\text { Tomar precauciones para mejorar la protección, } \\
\text { mantener, eliminar, controlar y minimizar las } \\
\text { posibilidades de que se produzca una mayor } \\
\text { exposición. } \\
\text { Establecer medidas adicionales para restablecer el } \\
\text { control en caso de que se produzca una situación de } \\
\text { alto riesgo, a pesar de las precauciones. }\end{array}$ \\
\hline $\begin{array}{l}\text { Posibles riesgos aunque no hay pruebas que vayan a } \\
\text { dar lugar a enfermedades o lesiones. }\end{array}$ & $\begin{array}{l}\text { Comparar las medidas actuales con las normas. Si la } \\
\text { comparación es desfavorable, determinar que debe } \\
\text { hacerse para mejorar las medidas de prevención y } \\
\text { protección. }\end{array}$ \\
\hline $\begin{array}{l}\text { Los riesgos se controlan adecuadamente pero las } \\
\text { actuaciones no se ajustan a la legislación vigente. }\end{array}$ & $\begin{array}{l}\text { Eliminar los riesgos o modificar el sistema utilizando } \\
\text { para controlarlos los principios establecidos. }\end{array}$ \\
\hline $\begin{array}{l}\text { Los riesgos son altos y no se controlan de manera } \\
\text { apropiada en la actualidad. }\end{array}$ & $\begin{array}{l}\text { Determinar y aplicar inmediatamente medidas } \\
\text { provisionales para impedir o controlar la exposición } \\
\text { a los riesgos. Considerar la posibilidad de detener el } \\
\text { proceso. Evaluar los requisitos a largo plazo. }\end{array}$ \\
\hline No hay ninguna prueba de existencia de riesgos. & $\begin{array}{l}\text { Seguir recabando toda la información hasta que } \\
\text { pueda llegarse a una de las conclusiones anteriores. } \\
\text { Mientras tanto aplicar los principios para reducir al } \\
\text { mínimo la exposición al riesgo. }\end{array}$ \\
\hline
\end{tabular}

Una vez realizada la evaluación de los riesgos laborales por ruido de acuerdo a los métodos propios de la entidad, comúnmente llamados tradicionales; se lleva a cabo la evaluación de acuerdo al método propuesto. 


\section{Método de evaluación teniendo en cuenta los niveles de seguridad}

El análisis de riesgos basado en la evaluación de la seguridad es un enfoque desarrollado en la actualidad, así como consideraciones en otras industrias y los diagramas de causa y efecto en cada proceso ${ }^{17}$.

El método propone diferentes pasos metodológicos para la evaluación de riesgos laborales por ruido, bajo el enfoque de defensa en profundidad de los niveles de seguridad (ENS), como se muestra en la siguiente figura:

Figura 1. Pasos para la evaluación de riesgos laborales por ruido

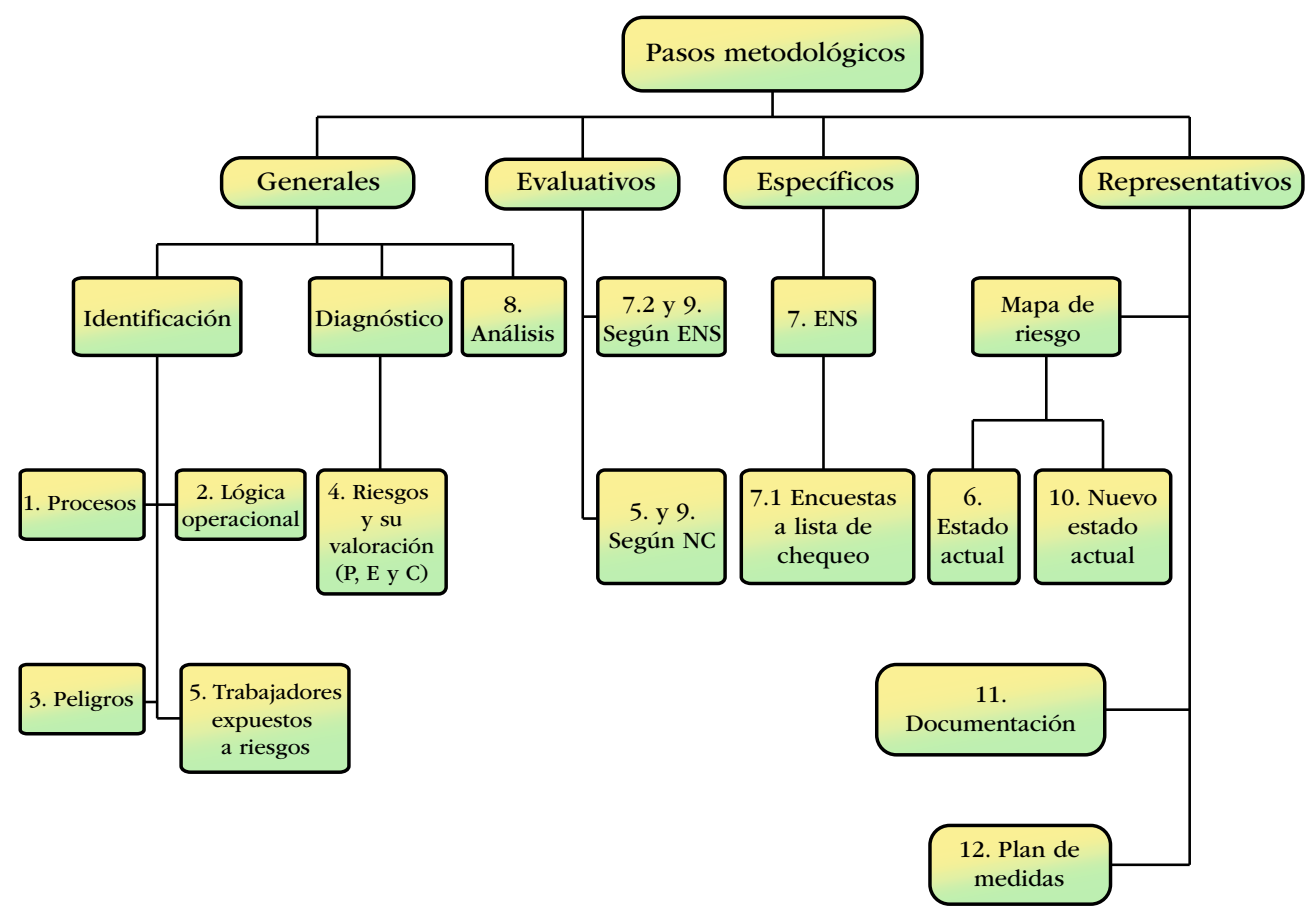

Los pasos de evaluación de riesgos laborales por ruido se estructuraron de acuerdo a las funciones fundamentales que se realizan, es decir generales, específicos, representativos y evaluativos.

Los pasos generales están formados por la identificación, diagnóstico y análisis, es decir, es la necesaria familiarización con la empresa objeto de estudio, teniendo en cuenta las interrelaciones y su incidencia en la salud ocupacional, empleando los mecanismos normativos.

Los pasos evaluativos están integrados por la evaluación de los riesgos laborales por ruido según las normas cubanas NC de ruido.

Los pasos específicos son los que aplican el método de evaluación de niveles básicos de seguridad (ENS) a los riesgos laborales por ruido

Los pasos representativos muestran de manera gráfica, los mapas de riesgo laboral y los monitores de riesgo. Además se incluyen los resultados de evaluación de los riesgos laborales por ruido de manera escrita, como evidencia.

El método para la identificación, evaluación y gestión de la prevención de los riesgos que afectan la seguridad y salud de los trabajadores, teniendo en cuenta los niveles básicos de seguridad (ENS), se realiza de acuerdo a los principios básicos de seguridad ${ }^{18}$ y con el enfoque de defensa en profundidad, ya que los niveles o escalones de seguridad, se estructuran de forma arbórea con cuatro niveles bien definidos, donde los niveles de defensa tributan al nodo del nivel superior de manera lógica. Este método se ha empleado 
en la evaluación de los riesgos tecnológicos en empresas de la aeronáutica y la petroquímica.

Los niveles o escalones de seguridad, se asocian al concepto de "barreras de seguridad" 19 cuya función general es planificar para prevenir, controlar, o mitigar la propagación de una condición o evento en condiciones no deseadas.

Para aplicar ENS, se determinan los aspectos de seguridad (AS) y los elementos que lo conforman, dentro de cada escalón de defensa, aplicables a los riesgos laborales en la CTE de 100 MW y se genera la base de conocimientos, clasificando en ítems positivos para la seguridad (S) o negativos (N); que se somete a evaluación. Posteriormente se introducen los AS y sus ítems, con su respectiva clasificación cualitativa (S) o (N), en la Hoja de Trabajo de ENS, se evalúan las listas, siguiendo los procedimientos prácticos del software ENS y se realiza el análisis e interpretación de resultados (primera evaluación con los primeros datos predeterminados en las bases de conocimiento y revisados por los expertos). Se procede a evaluar las listas por escalones y determinar el nivel de seguridad (ENS), a partir de los AS y sus ítems correspondientes y al análisis de resultados (listado de AS dominantes o sea, ordenados por importancia, para cada escalón de defensa dominante para la instalación o ENS), para la presentación de medidas correctivas para la mejora de la seguridad.

Para agilizar su ejecución se emplea la informática y se toman experiencias en otras ramas 20. Una vez determinados los aspectos de seguridad y conciliados con los especialistas, se aplican las potencialidades del software ASeC.

Para interpretar la seguridad de una instalación por niveles, se toma la altura como referencia, de modo que el nivel de seguridad es directamente proporcional a esta. A continuación se analiza a través del riesgo y la definición de las tres zonas generales de ENS, así como se explican los Niveles de Seguridad según ENS y la interpretación de cada uno de ellos, tomando como referencia la siguiente figura:

Figura 2. Zonas generales de ENS

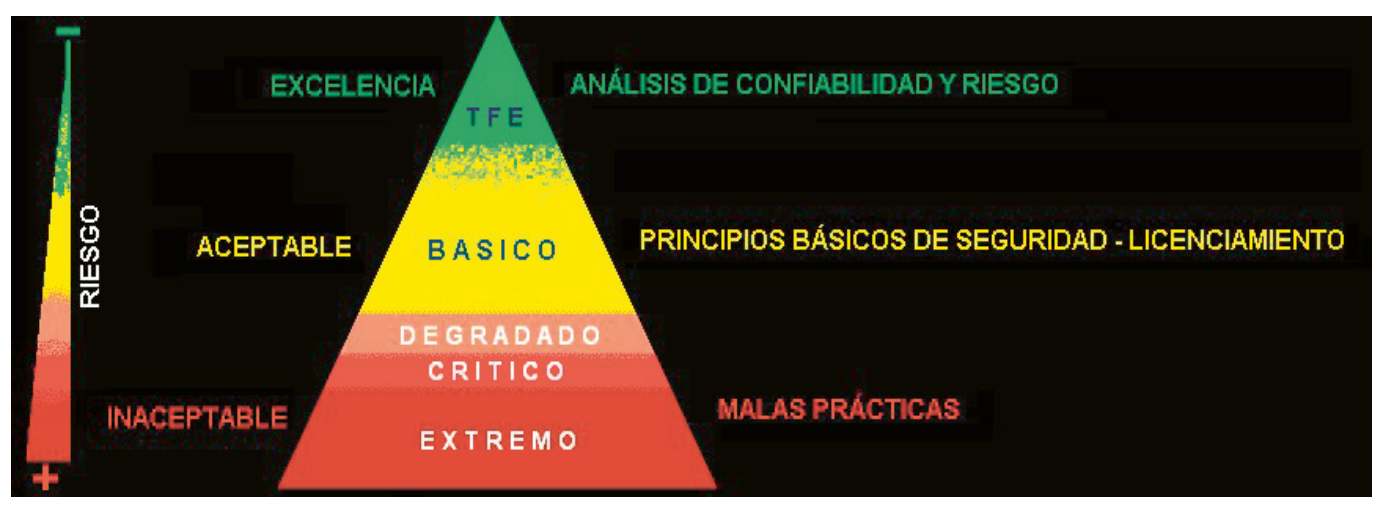

- Riesgo en la zona de TFE: Equivale al área que abarca la zona de color verde (mínimo).

- Riesgo en la zona Aceptable: Equivale al área que abarca la suma de las zonas verde y amarilla, por lo que es mayor que el riesgo en la zona TFE.

- Riesgo en la zona Inaceptable degradado: Equivale al área que abarca la suma de las zonas color verde, amarillo y rosado, por lo cual es superior a las dos anteriores.

- Riesgo en la zona Inaceptable crítico: Equivale al área que abarca la suma de las zonas anteriores más el área de la zona de color rojo.

- Riesgo en la zona Inaceptable extremo: Equivale al área que abarca a todas las zonas. 


\section{Interpretación de las zonas de riesgo (seguridad)}

ENS parte de una escala numérica, que se hace corresponder con la escala cualitativa que representa el triángulo de la figura 2. La explicación de las zonas de riesgo en función de la escala cualicuantitativa se presenta a continuación.

\section{- Nivel INACEPTABLE: ENS >15\%}

Se han comprobado deficiencias o debilidades importantes en la defensa en profundidad de la instalación objeto de estudio, tras la evaluación de la aplicación de los principios fundamentales de seguridad, que garantizan el buen estado es esta. Esas debilidades deben ser erradicadas antes de realizar cualquier otro estudio de mayor profundidad, basado en técnicas de análisis de riesgo o confiabilidad de mayor complejidad. Este estado significa que no podrá funcionar correctamente la Defensa en Profundidad, ya sea porque no se han concebido correctamente en el diseño o porque su estado se ha deteriorado durante la operación por malas prácticas. Las medidas que conllevan a una correcta aplicación de los principios afectados elevarán el nivel de seguridad a un nivel ACEPTABLE o BÁSICO, disminuyendo así la probabilidad de ocurrencia de accidentes. Este nivel se subdivide en tres zonas para mejorar el proceso de toma de decisiones concernientes a la seguridad, a saber, INACEPTABLE-Degradado $(15 \%<$ ENS $\leq 25 \%)$; INACEPTABLE-Crítico $(25 \%<$ ENS $\leq 35 \%)$; INACEPTABLE-Extremo (ENS $>35 \%$ )

\section{- Nivel ACEPTABLE o BASICO: $5 \%<$ ENS $\leq 15 \%$}

No se han detectado desviaciones, debilidades o insuficiencias importantes en la aplicación de los principios básicos de seguridad. Ello asume que se ha asegurado que el funcionamiento de la instalación no causará daño al personal, a la población circundante y al medio ambiente, así como que los accidentes base de diseño han sido tenidos en cuenta y por lo tanto serían controlados en caso de ocurrir, sin que ello conlleve a daño al personal, la población y el medio ambiente circundantes (instalación con licencia o algún tipo de permiso reglamentario para ejecutar la etapa del proyecto actual). No se incluye en este nivel el control de los accidentes fuera de las bases de diseño (accidentes severos).

\section{- Nivel de EXCELENCIA lo Tendencias Favorables a la Excelencia): $0 \% \leq$ ENS $\leq 5 \%$}

Representa el nivel de seguridad más alto que es razonablemente factible alcanzar o nivel de riesgo más bajo que es razonablemente factible alcanzar (principio ALARP 21 aplicado en la normativa británica de seguridad y salud laboral y en la aviación civil), a partir de aplicar técnicas especializadas de evaluación de la seguridad, que permiten de una manera justificada determinar aquellos puntos débiles difícilmente visibles a través de los medios tradicionales de evaluación de la seguridad de una instalación, que se encuentre en el nivel Aceptable o Básico. Estar en el nivel matizado por las Tendencias Favorables a la Excelencia (TFE) significa que se han minimizado las oportunidades para la ocurrencia de eventos previsibles, lo que implica que la probabilidad de accidentes será aún más baja que la que se puede lograr en el nivel básico, de modo que el riesgo inducido por la explotación del objeto industrial sea despreciable. Las industrias con riesgo potencial asociado a su explotación deben proponerse como meta alcanzar este nivel, pues tienen peligros asociados de alcance mayor, pudiendo afectar a toda la instalación dentro de los límites del emplazamiento o sus alrededores.

De manera resumida se muestra en la siguiente figura el método propuesto: 
Figura 3. Método para evaluar riesgos laborales por ruido

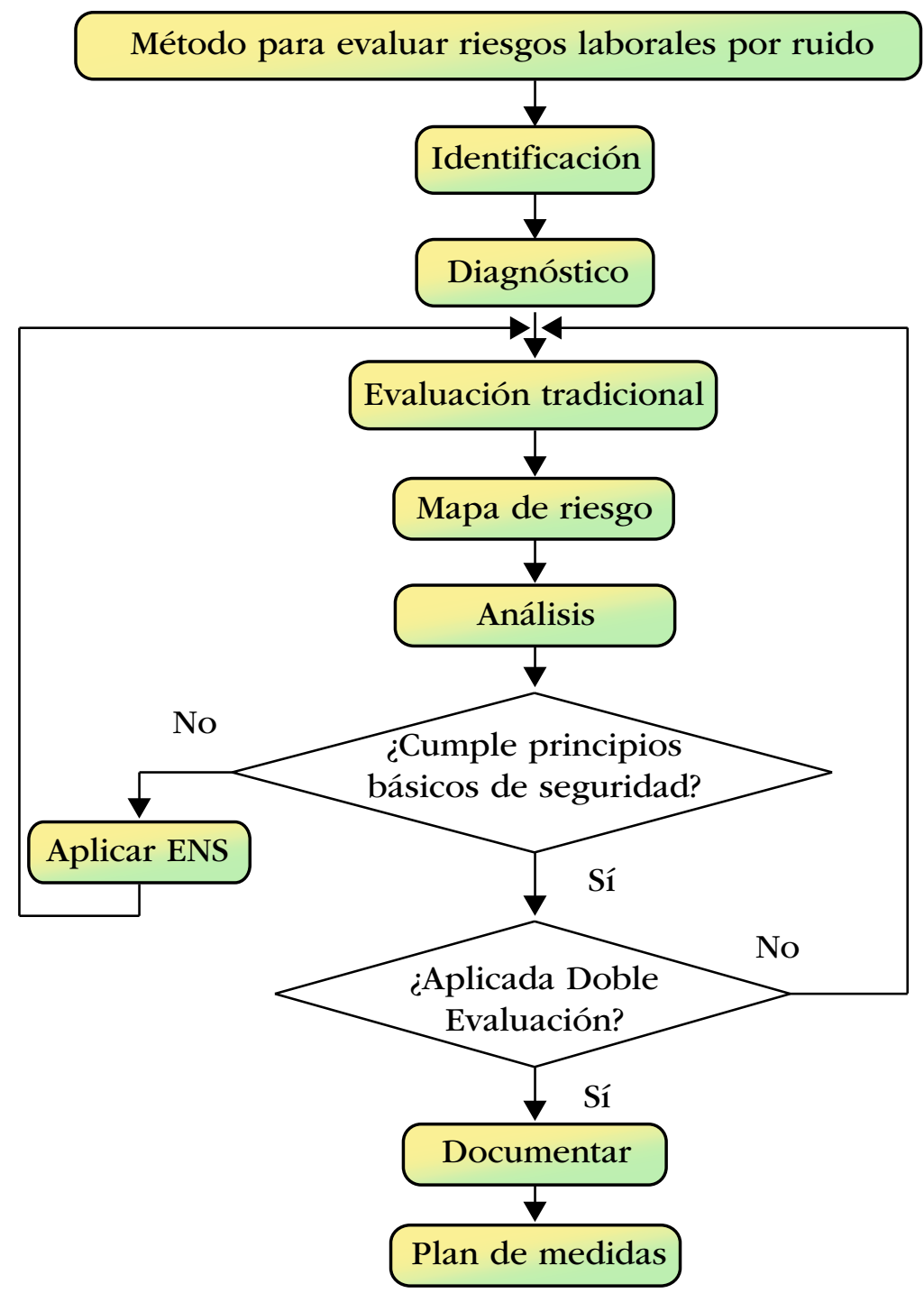

Como se observa, los pasos metodológicos propuestos requieren la inclusión de los métodos tradicionales que se aplican en la CTE, para evaluar los riesgos laborales por ruido; y el método de evaluación de riesgos basado en los niveles básicos de seguridad, se insertó como parte de los pasos metodológicos del método propuesto.

En la representación gráfica se estableció un código de colores para identificar dentro del método propuesto, qué pasos corresponden al método tradicional y cuál al ENS; así como el nivel de incidencia de cada uno de ellos.

\section{RESULTADOS}

Una vez aplicada la evaluación por las tres metodologías, se hace necesario analizar de manera detallada cada uno de los resultados para valorar su viabilidad y pertinencia.

\section{Resultados de la Evaluación de los riesgos para le implementación del Sistema de Gestión Ambiental en las entidades de la UNE}

Al aplicar este método se determinó que la generación de ruidos no es un aspecto significativo a tener en cuenta. 
En el inventario de ruido, se señala el local o instalación y las actividades o procesos en los cuales se produce, la evaluación de acuerdo a los criterios de severidad, frecuencia, efecto sobre la comunidad o los trabajadores y si está sujeto a regulación y su evaluación total.

\section{Resultados de la Evaluación de los riesgos de acuerdo al Manual de Procedimientos Seguridad y Protección}

De acuerdo a este método se identificaron los riesgos por ruido que pueden existir por áreas y de acuerdo a las actividades que se realizan.

Posteriormente se realiza el análisis de acuerdo a la Legislación:

En el estudio de ruido en la Central Termoeléctrica de 100 MW los resultados de las mediciones, de acuerdo a las normas, evidenciaron una dispersión del nivel sonoro, con tendencia estable a los valores máximos en determinadas áreas según la NC de ruido.

Para contrarrestar el ruido, se emplean los dispositivos de protección personal, tapones o ambos simultáneamente, según sea la intensidad, las frecuencias y el tiempo de exposición al ruido.

\section{Resultados de la Evaluación de los riesgos de acuerdo al Método de Evaluación de los Niveles de Seguridad.}

Producto de la estructuración del método ENS y la escala numérica asociada, los resultados globales permiten conocer, no solamente el perfil de riesgo de la instalación, sino la jerarquización de los contribuyentes al mismo.

La importancia de esta potencialidad radica en que se facilita el proceso de toma de decisiones, dentro de la gestión de la seguridad, al orientar a la gerencia sobre cuáles son sus prioridades de atención para una mejora de la seguridad, tomando en consideración criterios económicos.

Los aspectos de seguridad evaluados negativamente son los que contribuyen a la degradación de la seguridad del objeto industrial. Los mismos sirven de apoyo a la toma de decisiones, teniendo en cuenta el grado de importancia de la influencia negativa de cada aspecto de seguridad evaluado dentro de cada escalón.

Para elevar el nivel de seguridad del AS, se requieren tomar medidas correctivas dirigidas a modificar los ítems calificados negativamente a positivos.

\section{DISCUSIÓN}

Los resultados de la aplicación de los métodos para evaluar los riesgos laborales: Evaluación de los riesgos para la implementación del Sistema de Gestión Ambiental en las entidades de la UNE y Evaluación de los riesgos de acuerdo al Manual de Procedimientos de Seguridad y Protección, permitieron determinar las zonas donde los riesgos estaban por encima de los parámetros aceptables, pero no establecen las prioridades de las medidas correctivas para su disminución.

El método propuesto incluye la doble evaluación: tradicional y aplicando ENS; por lo que los especialistas pueden emprender acciones tanto correctivas como preventivas, ya que una potencialidad fundamental de la aplicación del ENS es precisamente que se anticipa al problema. En el caso de los métodos tradicionales, donde se toman en cuenta los rangos normativos, solo se puede mitigar o contrarrestar el riesgo laboral por ruido al detectarse lugares y trabajadores expuestos en esa zona que no cumplen las normas. El ENS establece múltiples barreras para evitar que ese riesgo se materialice, teniendo en cuenta además no solo las condiciones de trabajo normales y las anormales. 
Las ventajas evidentes de los otros métodos no se comparan con respecto a la gestión de la seguridad, ya que no tienen en cuenta el análisis de seguridad que se realiza empleando ENS, que es un sistema lógicamente estructurado, bajo los principios básicos de seguridad, que estructura por escalones de defensa y retoma los años de experiencia en seguridad en empresas de alto riego y disponibilidad. Existen otros métodos que son de penalización, de evaluaciones comparativas, pero en ellos no se realiza la estructuración lógica desde el pensamiento de la defensa en profundidad.

La aplicación de ENS en el método de evaluación de los riesgos laborales hace la función de complementar el método, mientras que en los casos de bioseguridad en laboratorios la función es la de integrar los métodos existentes; y para los riesgos tecnológicos el método es abarcador.

\section{CONCLUSIONES}

1. Se diseñó un método de evaluación de los riesgos laborales por ruidos en la Central Termoeléctrica de $100 \mathrm{MW}$.

2. Los resultados al aplicar el método de evaluación por niveles de seguridad muestran que los registros y aspectos de seguridad más importantes fueron exhaustivamente identificados por su nivel de contribución.

3. La evaluación empleando escalones de defensa en profundidad aplicado a los riesgos laborales posibilita un análisis con el enfoque de procesos, y estimar la contribución relativa y sus causas.

4. La identificación de los aspectos de seguridad evaluados de negativos que más dominan el riesgo, permite tomar las medidas correctivas sobre sus causas, y resolverlas en un corto plazo.

5. La creación de las nuevas listas de chequeos genéricas con el análisis de sus dependencias funcionales teniendo en cuenta los riesgos laborales por ruido, contribuye al perfeccionamiento y ampliación de la versatilidad de las opciones de evaluación del software ASeC y su extensión conjugada a otros parámetros de trabajo dentro de este campo.

\section{REFERENCIAS BIBLIOGRÁFICAS}

1. OSHA 29 CFR PART 1910 [Consulta: enero de 2015]; Disponible en: http://www.osha.gov/pls/oshaweb/ owasrch.search_form?p_doc_type=STANDARDS\&p_toc_level=1\&p_keyalue $=1910$.

2. OFICINA NACIONAL DE NORMALIZACION Seguridad y Salud en el Trabajo - Sistema de Gestión de Seguridad y Salud en el Trabajo — Requisitos. NC18001: 2005 (ICS: 13.100) La Habana: ONN, 2005. 25 p.

3. CUBA, MINISTERIO DE TRABAJO Y SEGURIDAD SOCIAL, "Resolución 23/97 del MTSS Metodología para la Identificación, Evaluación y Gestión de la Prevención de los Riesgos que afectan la Seguridad y Salud de los Trabajadores" Gaceta Oficial de la República de Cuba. Edición Ordinaria. La Habana, 12 de febrero de 1998, No. 9, pp. 5.

4. Case K, Norb A, Chow P. "A diagnostic service tool using FMEA" en International Journal of Computer Integrated Manufacturing Vol. 23, No. 7, July 2010, 640-654 ISSN 1362-3052.

5. M. Casamirra, F. Castiglia, M. Giardina, C. Lombardo "Safety studies of a hydrogen refuelling station: Determination of the occurrence frequency of the accidental scenarios" en International Journal of Hydrogen Energy ISSN: 0360-3199 [Consulta: marzo de 2013]; Disponible en: http://www.sciencedirect. com/science/article/pii/S0360319909001505].

6. Arabian-Hoseynabadi H,. Oraee H, Tavner P. J. "Failure Modes and Effects Analysis (FMEA) for wind turbines" en: Electrical Power \& Energy Systems ISSN: 0142-0615 [Consulta: enero de 2013]; Disponible en: http://www.sciencedirect.com/science/article/pii/S0142061510000281.

7. Estorilio C., Posso R. K. "The reduction of irregularities in the use of "process FMEA" en: International Journal of Quality \& Reliability Management ISSN: 0265-671X [Consulta: enero de 2013]; Disponible en: http://www.emeraldinsight.com/journals.htm?articleid=1864948. 
8. Bachmann, V., Messnarz, R. "Improving safety and availability of complex systems by using an integrated design approach in development" en Journal of Software: Evolution and Process 2013; 25:341-349 Published online 31 January 2012 in Wiley Online Library (wileyonlinelibrary.com). DOI: 10.1002/smr.580.

9. Liin Rossinga, N., Lindb, M., Jensenc, N., Bay Jørgensena, S. "A functional HAZOP methodology" en: Computers and Chemical Engineering ISSN: 0098-1354 [Consulta: enero de 2013]; Disponible en: http:// www.sciencedirect.com/science/article/pii/S0098135409001793.

10. Dunjóa, J., Fthenakisb, V., Vílcheza, J. A., Arnaldos, J. "Hazard and operability (HAZOP) analysis. A literature review" en: Journal of Hazardous Materials ISSN: 0304-3894 [Consulta: enero de 2013]; Disponible en: http://www.ncbi.nlm.nih.gov/pubmed/19733970.

11. Cagno, E., Caron, F.,. Mancini, M. "Risk analysis in plant commissioning: the Multilevel Hazop" en Reliability Engineering and System Safety ISSN: 0951-8320 [Consulta: diciembre de 2012]; Disponible en: http://www.sciencedirect.com/science/article/pii/S0951832002000649.

12. Avila, S. F., Pessoa, b, F. L. P., Andradec, J. C. S "Social HAZOP at an Oil Refinery" en Wiley Online Library (wileyonlinelibrary.com) Process Safety Progress (Vol.32, No.1).

13. Markowski, A. S., Sam Mannan, M "ExSys-LOPA for the chemical process industry" en: Journal of Loss Prevention in the Process Industries ISSN: 0950-4230 [Consulta: diciembre de 2013]; Disponible en http:// www.sciencedirect.com/science/article/pii/S0950423010000525.

14. Cui, L. Shu, Y., Wangb, Z., Zhao, J., Qiu, T., Sun, W., Wei, Z. "HASILT: An intelligent software platform for HAZOP, LOPA, SRS and SIL verification" [Consulta: marzo de 2013]; Disponible en: http://dx.doi. org/10.1016/j.ress.2012.06.014

15. Imran Rashid, M., Ramzan, N., Iqbal, T,. Yasin, S., Yousafb, S. "Implementation Issues of PSM in a Fertilizer Plant: An Operations Engineer's Point of View "Process Safety Progress (Vol.32, No.1) Published online 30 January 2013 in Wiley Online Library (wileyonlinelibrary.com). DOI 10.1002/prs.11553 [Consulta: marzo de 2013].

16. Perdomo, M; Salomon, J; et. al. ASeC, An Advanced System for Operational Safety and Risk Assessment of Industrial Facilities with High Reliability Requirements. Rio Oil And Gas 2010. Expo and Conference. Rio de Janeiro, September 2010 Publicado nos Anais da Rio Oil \& Gas Expo and Conference 2010. Copyright 2010, Instituto Brasileiro de Petróleo, Gás e Biocombustíveis -IBP3090_10.

17. Mohammad D. AL-Tahat $\bigotimes$, Abdul Kareem M. Abdul Jawwad, Yousef L. Abu Nahleh "Ordinal Logistic Regression Model of Failure Mode and Effects Analysis (FMEA) in Pharmaceutical Tabletting Tools" en: Engineering Failure Analysis [Consulta: diciembre de 2013]; Disponible en: http://dx.doi.org/10.1016/j. engfailanal.2012.08.017

18. SALOMÓN, J Perdomo, M Análisis de Riesgo Industrial 2. Edición Adaptada de manera digital. Venezuela: Centro de Estudios Gerenciales ISID. Empresa de la Fundación Educativa "María Castellanos", 2001, pp.207, ISBN 980 00-1491-8.

19. Duijm, N. J., Markert, F. "Safety-barrier diagrams as a tool for modelling safety of hydrogen applications" en International Association for Hydrogen Energy. 34 (2009) 58 2-5868 [Consulta: marzo de 2014]; Disponible en: http://www.sciencedirect.com/science/article/pii/S0360319909002006.

20. J. Verde, Perdomo M., Salomón J "Aplicación de la Evaluación del Nivel de Seguridad en Instalaciones Petroleras”. VI Conferencia Internacional de las Ingenierías Mecánicas, Eléctricas e Industrial. UPADI, 2012. ISBN 978-959-247-094-1. La Habana, 9-13 de abril de 2012.

21. Rusli, R., Mohd Shariff, A. "Qualitative Assessment for Inherently Safer Design (QAISD) at preliminary design stage" en: Journal of Loss Prevention in the Process Industries 23 (2010) 157-165 [Consulta: enero de 2014]; Disponible en: http://www.sciencedirect.com/science/article/pii/S0950423009001132. 